\title{
Empathic Design: Cases Study of Designers Creating Empathic Phenomenons and The Dimensions of Empathic Design
}

\author{
Shu-huei Wang \\ MingDao University, ChangHua,Taiwan
}

\author{
Shyh-huei Hwang \\ National Yunlin University of Science and Technology, \\ Yunlin, Taiwan
}

\begin{abstract}
Ickes (1997) has mentioned that empathy is a complex form of psychological inference in which observation, memory, knowledge, and reasoning are combined to yield insights into the thoughts and feelings of others. The phenomenon of empathy has been discussed in philosophy, aesthetics, and psychology for many years. In the beginning of the 20th century, an extensive discussion on this phenomenon has been led by European intuition phenomenologists such as Husserl, Sartre, Brunswik, and Heider, etc. It is known that the theory of its development relates to understanding human feelings and emotions. Empathic design in the design field has developed emotional design, inclusion design and universal design, etc. Empathic design is an emerging issue in the design field. What are empathic design, designing process, design methodologies, and a strategic design model? Why is empathic design important to the creative and designing processes? Empathic design is one of the useful ways to understand interpersonal relationships that might influence the creative and designing processes, design strategies and innovation. Empathic design that emphasizes listening to the voice from customer's heart and designing this into design has been proved to be something the customer really needs in brand communication messages and brand values. This paper researches, investigates and gathers the research methodologies and combines a literature review, case study, structured interviews, and grounded theory. The structured interviews comprise four designers who worked in the brand design department, including two senior designers and two junior designers. Thus, a literature review is conducted in this research to discuss the empathic design model, explore founding theories and then seek to analyze and propose a structured empathic design model. Finally, the research presents an empathic design model to explain several denominations, which are empathic communication, empathic aesthetics, empathic innovation, empathic methodologies, empathic culture and others. The design creation process from abstract ideas to concrete manifestation for reference of design students, design teaching, and design practice.
\end{abstract}

Keywords: empathic design, design methodologies, empathy, design processes, empathic design model

\section{Introduction}

The characteristics of design in the 21st century are to meet the emotional needs of modern consumers' hearts and to make them touched and experience something. A study on empathic design is to explore feeling into,

Shu-huei Wang, assistant professor, Department of Digital Design, MingDao University.

Shyh-huei Hwang, professor, Graduate school of Design, National Yunlin University of Science and Technology. 
creation and understanding of designers towards end-users. The essential of an empathic design is to get in touch with real end-users with empathy to satisfy their requirements and solve their problems. Designers have to investigate experiences of end-users in a systematic and empathic way. Psychological analogies of empathy towards objects, aesthetics, environment, and culture are manifested by association, projection, and inner imitation, etc. for immediate and efficient understanding. However, how can designers grasp the true demands of end-users, those not perceived by end-users, either in particular? A case study, an in-depth interview, the design processes of four brand designers, literature review and the grounded theory are used in this research to summarize the dimensions of empathic design. The design creation process from abstract ideas to concrete manifestation for reference of design students, design teaching, and design practice.

\section{Literature Review}

Dorothy and Rayport, professors of Harvard Business School, proposed Empathic Design (1997). They considered designers needed to exercise empathy by observing the environment of consumers, acquired the ability of feeling for consumers and designed the things that met their real needs. How can designers develop empathy for empathic design, how can empathic design satisfy the "hidden" needs of end-users and how can originality and process of design have better performance through empathic design are the issues for study.In other words, what are the designer of the design process, design methods, and design steps? How designers think the position of standing customers real needs? Finally, this model can provide designers, students or design of the interest can refer to the application.

\section{The Phenomenon of Empathy}

The phenomenon of empathy: (1) When we watch a movie, are absorbed in the plot and shed tears because we are touched by the performance of a certain personage in the play (to feel indebted as if it were received in person), that is "empathy". (2) When we enjoying of art works and empathize with them, we have feelings towards the scenes depicted in the paintings (to identify with), which is the so-called "empathy". (3) When we listen to music and integrate with the melody, our emotions vary with the tempos of music (fantasies of happiness, anger, sadness and joy) causing "empathy". (4) As we listen to the misfortunes of others, we understand their feelings like we used to go through them (to feel for others), which is "empathy". There are many of other examples of "empathy" and application of empathy to design (see Figure 1).

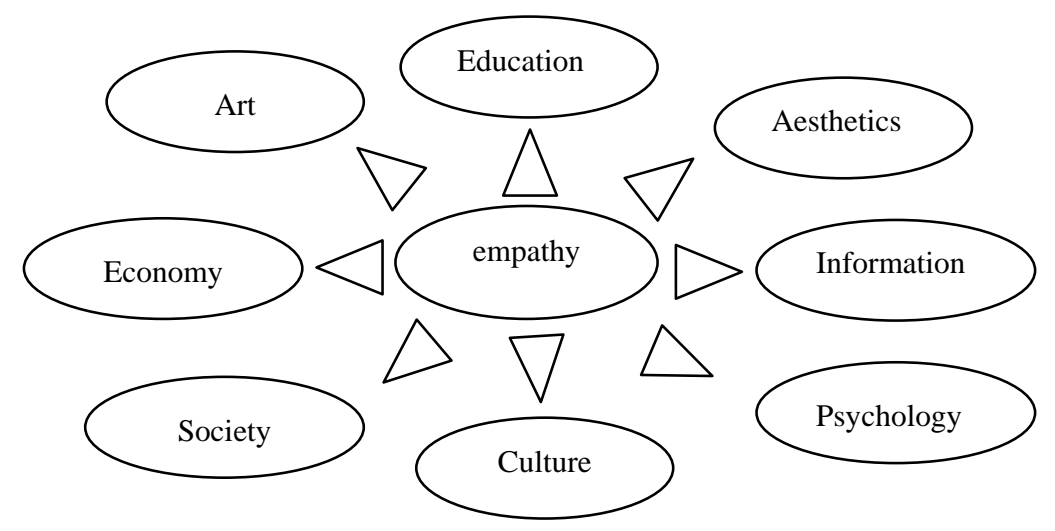

Figure 1. Empathy is wide fields of study (this research illustration). 


\section{Empathic Design}

The empathy reveals the transitional interpretations of from philosophy, aesthetics, and psychology to the extended application in the field of design currently. The article entitled Empathic Design vs. Empathetic Design: A History of Confusion authored by Landwehr (2007). He located 12,700 pieces of information on empathic design through Google and indicated empathic design was first defined by Leonard and Raport in 1997 claiming how designers could know the potential requirements of users and it was a procedure of a design method. Nevertheless, empathic design is not merely a design approach; on the contrary, a contextual understanding of the nature of empathy itself and the evolutions of empathic design is required.

In 1997, Will Ickes proposed four stages of empathy in his book, including judging the other party, mutual understanding, accuracy and sensibility and precise deduction. Koskinen, Battarbee, and Mattelmäki, in 2003 considered designers needed to use systematic ways to study experiences and such ways had to be "empathic" for an in-depth understanding of the users. In 2004, Feng, Lazar, and Preece presented the accuracy of empathy and claimed empirical studies emphasized empathy and the interpersonal trust conveyed by the online real time messages. In 2005, Kleef, Trijp, and Luning deemed empathic design was a feasible way for market surveys on the early stage. Empathic design is classified as an approach for early product R\&D by 10 research methods. In 2005, Chapman proposed the endurance of empathy from the perspective of purchase; nevertheless, the lifespan of a product is based on the endurance of empathy given by the product in this respect. In 2007, Fritsch, Júdice, Soini, and Tretten thought empathy was a true understanding of others' experiences as well as an approach for simulating, creating, and communicating mental images.

At present, several major issues of empathic design can be concluded as follows: (1) design methods; (2) the contexts applied (contextual design); (3) innovation and originality (innovative design); (4) listening to the voices of customers (user design centered); (5) endurance; (6) understanding and accuracy; (7) simulating, creating, and communicating mental images (a real understanding of others' experiences); (8) feeling indebted as if it were received in person (to feel for others); (9) design empathety; and (10) online empathic communication. However, these are western views on empathic design. In fact, they are different from oriental perspectives to some extent. Main points of empathic design will be summed up in the next section.

\section{The Summaries of Empathic Design}

Empathic design is emerging in the field of design and developing from different perspectives in the east and the west. The thread of thought and the knowledge system of empathic design are constructed and based on philosophy, aesthetics, and psychology, etc. Accordingly, empathic design can not only be applied to design, but also to the process of creation, a communication medium and experience of spatial display:

(1) Empathic design during the process of creation: The inner simulation and projected unity of object and subject of empathy imply the integration of the self and the object to become as one. For example, designers may exercise empathy like placing themselves in others' position, identifying with customers and feeling for them to design the products that truly meet the demands and deeply touch the minds, souls, bodies, and memories of customers with tangible or intangible messages understood by designers.

(2) Empathic design as a medium for communication: Speroff (1953) considered that empathy was a capability of putting oneself into another's shoes to establish a relationship and experience the feelings, reactions 
and behavior of others. Susceptivity and understanding are required for human communication. Therefore, people can understand and experience situations each other. On the other hand, Feng, Lazar, and Preece (2004) proposed the accuracy of empathy and deemed empirical studies stressed empathy and the trust among people when sending online messages. Technological media have been applied to empathic design for investigating issues related to online users for the past few years. Discussion keeps growing, which may be due to extensive application of new technologies.

(3) Empathic design for experiencing spatial exhibitions: The aesthetic experience of the observer is mainly based and the aesthetics perceived is emphasized like a piece work of art as a significant form. According to Preece, we may comprehend self and object pleasures perceived when people appreciate art or go through an aesthetic experience are self emotional projection actually. Consequently, as people appreciate works of art or have an aesthetic experience, interaction between the artists and the observers occurs through the works.

(4) Systematic way of empathic design: Dorothy and Rayport, professors of Harvard Business School, proposed the aspect of empathic design as well as the method and the design process to understand the real needs of users. They also presented five steps for empathic design. Koskinen, Battarbee, and Mattelmäki (2003) deemed designers had to study experiences in asystematic way, which should be empathic for a (in-depth) understanding of users. Wikipedia (2008) ${ }^{1}$ defined empathic design as an approach to design where researchers or developers try to get closer to the lives and experiences of (putative, potential, or future) end-users. In sum, empathic design is also a design method for early product development as well as innovation. Take IDEO, a design company, as an example. They use a similar design process and develop great originality to serve customers.

(5) Dorothy and Rayport, identify the five key steps in empathic design as 1) customer observation; 2) data gathering through visual; 3) auditory and sensory cues; 4) analysis of data; and 5) brainstorming, and the development of prototypes of possible solutions.

\section{Methodologies}

Four designers of well-known brands are selected and their design processes are compared. An in-depth interview is conducted and various methods, sources, and theories are adopted for a cross comparison. The grounded theory is applied to analysis. Finally, dimensions of empathic design are concluded.

\section{Research Method}

Sources of information collected for this research are from in-depth interviews with four designers, literature review and comparisons of various case studies. Cross reference and verification are made to confirm the consistency of data.

\section{Research Structure}

The structure of this research is based on issues related to empathic design, literature review, comparisons of case studies of design processes for four designers of the same brand and analysis of in-depth interviews through the grounded theory for dimensions of empathic design as illustrated in Figure 2.

\footnotetext{
${ }^{1}$ Wikipedia Retrieved June 30, 2008, from http://en.wikipedia.org/wiki/Empathic_design.
} 


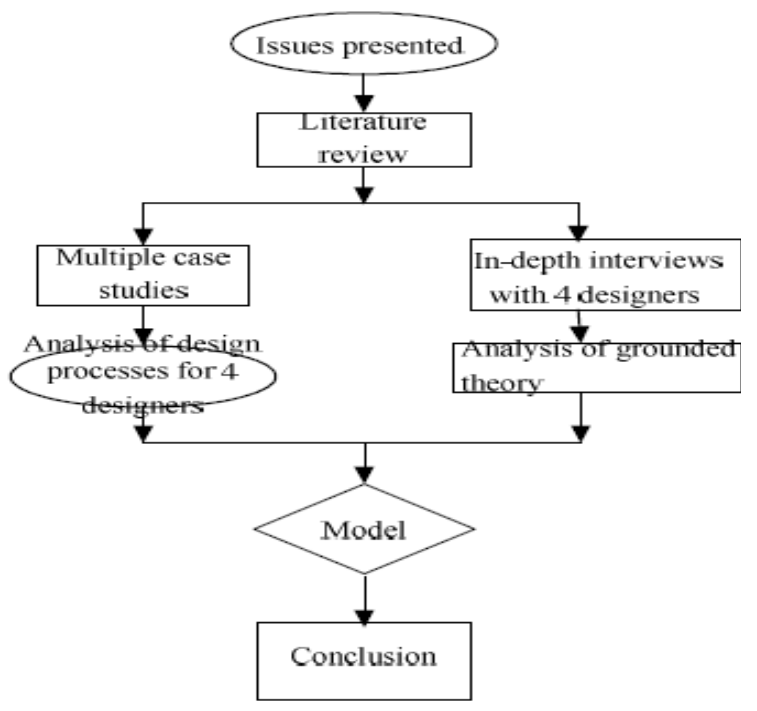

Figure 2. Structure of study (this research illustration).

\section{Participants}

Four brand designers accepted interviews and their basic information are listed in Table 1 . The question items of the interview are described included: (1) How do you reflect your idea on design? Please, to described your design processed. (2) How do you understand the needs of users during the changeable design process? etc. (see Table 1).

Table1

Basic Data of Object Under Study (This Research Illustration)

\begin{tabular}{|l|l|l|l|l|}
\hline No. & P1 & P2 & P3 & P4 \\
\hline Gender & Male & Female & Male & Female \\
\hline Age & 30 & 40 & 50 & 48 \\
\hline Educational background & University & University & Senior high school & University \\
\hline No. of service design & 5 years & 4 years & 26 years & 24 years \\
\hline Project design & Counter design & Counter design & Counter design & Counter design \\
\hline Date of interview & 2008.9 .15 & 2008.9 .15 & 2008.9 .15 & 2008.8 .15 \\
\hline Responsible brand channel & Watsons & Watsons & Specialty shop & Department store \\
\hline Design experience & 6 years & 17 years & 26 years & 27 years \\
\hline
\end{tabular}

\section{Data Gathering}

Information of this research is gathered by interviews mostly. In-depth interview is a way of gathering data that has existed for a long time (Platt, 2002) and is also the most frequently used method for qualitative researches (Marshall \& Rossman, 1995). The details of design processes for four designers are collected by tape recording and in-depth interviews.

\section{Results and Discussions}

A well-known cosmetics brand is used for case study and designers of this brand are served as the objects of study. Case studies were conducted on these four designers and their design projects were given as examples. 


\section{Analysis of Case Studies}

The design process, ideas, and works of these four individual designers are described and summarized.

(1) Case designer P1.

I would discuss with my superior first about any conditions (limits) involved at work. Changes are made easily to cope with the ideas that pop up to the superior suddenly causing a failure of the effect expected from time to time. I have to grasp the features of my own works. Finally, I need to explain the original concept of the work. Forms can change, but not the characteristics since such features enjoy the uniqueness of the brand and the brand concept.

(2) Case designer P2.

Features and demands of an express shop and a specialty shop are different. I consider the traits, function, integrity, market characteristics, integration and development of company regulations. For the channel of specialty shops, standards and the system are specified. Functional adjustment like "open window" is necessary for design of new concepts. The counter brand is different from other self-selective brands. Customer requirements and the philosophy of the company should be satisfied as well.

(3) Case designer P3.

First of all, you have to know what you do. Designers of enterprises are confronted by proprietors or users. You need to make sure of your position, investigate and measure the nearby environment, the general status in downtown, geographical conditions, the location of the shop and its specific requirements. Meanwhile, you have to verify user needs. The process of design is site survey, an understanding of shop requirements, measurement and verification of user needs.

(4). Case designer P4.

Information is collected in compliance with customer requirements and the subject matter is established. In addition, personal opinions and views are added. Consequently, the overall design process includes customer requirements, data gathering, information collected according to the subject matter and addition of personal opinions. Take department stores as an example. Areas and functions of current counters are compared and adjusted in accordance with the existing market in Taiwan. A space for one-on-one service is provided to customers for more diversified and private service.

Three of them regard they accomplish customer satisfaction by experience in person and thinking in customer's shoes. They reason by analogy; i.e., they deem themselves as users and experience the overall purchase process so that they may obtain new ideas or modify from such experience.

\section{Analysis of Grounded Theory}

Open coding means to retrieve the terms (schemas) related to the subject from the information collected during in-depth interviews and condense these schemas to several categories, which are the ideas or the subject matter (cognitive structure) representing the special significance symbolized by the information. The purpose of axial coding is to categorize the ideas (or subject matters) located from open coding for a more precise and complicated explanation of a certain phenomenon and completion of axial concepts (Herzog, 1996). Case interviews with four designers were conducted and research theories related to empathic design were integrated resulting in 7 dimensions, 152 conceptions, 22 open codes, and 7 categories (see Table 2). 
Table 2

Summarized Coding Table (This Research Proposes Grounded Theory Analysis Results)

\begin{tabular}{|c|c|c|c|}
\hline Category & Open coding & Category & Open coding \\
\hline \multirow{3}{*}{ 1. Communication } & Desigh communication process & \multirow{3}{*}{ 5. aesthetics } & Professional aesthetics \\
\hline & Reach a common consensus & & Fulfill aesthetics \\
\hline & Brand communication & & Same position \\
\hline \multirow{4}{*}{ 2. experience } & Experience process & \multirow{4}{*}{ 6. Innovation } & Breakthrough of old thinking \\
\hline & Role-play & & Innovative performance \\
\hline & Incorporate requirement & & Innovatiove design \\
\hline & Reality drill & & Integrate with the environment \\
\hline \multirow{4}{*}{ 3. methodologies } & Design process & \multirow{4}{*}{ 7. Culture } & Brand culture \\
\hline & Market research & & Cultural background \\
\hline & Understand customer needs & & Service quality \\
\hline & Experience in person & & \\
\hline \multirow{2}{*}{ 4. Creativity } & Design thinking & & \\
\hline & Creative ways & & \\
\hline
\end{tabular}

(1) empathic communication.

Immanuel Kant deemed that the foundation of resonance was a common feeling; i.e., everyone perceived in common. Rifykin pointed out that humankind sought after a common consensus through empathy, an ability of experiencing the feelings of others (Rifykin, 2001). A common view is based on communication, which refers to a process of sending, receiving, and understanding messages. Effective communication is required for each dimension to achieve the expected result for the design process in particular. A designer extends his/herideas from the theme.

keeps communicating and modifying and reaches a common view with the proprietor at last. Brand messages and features are included on (p1-1-3). Combination of corporate regulations and implementation of ideas are on (P2-1-2).

(2) empathic experiences.

Speroff (1953) considered empathy was a capability of putting yourself in another's shoes, establishing a relationship and experiencing another's feelings, reactions, and behavior. According to Hogan (1969), empathy was to understand other's mental situation or status intellectually or imaginatively without really experiencing their feelings. The experiencing process,

includes counting yourself as a customer to experience (P4-5-1), role-play, thinking in customers' or demanders' shoes (P2-7-1)

incorporation of requirements—designers need to incorporate proprietor's needs (P3-7-3) and having a better understanding of customer needs through real drills (P2-5-2).

(3) empathic methodologies.

Leonard and Rayport proposed five steps for the process of an empathic design. Koskinen, Battarbee, and Mattelmäki (2003) deemed designers required systematic ways to study experiences and these ways should be empathic for an in-depth understanding of users. The breadth of design studies and experiences:

surveys of customer needs, data gathering, information collected based on themes and incorporation of personal opinions ( P4-1-5). Local designers integrate market survey results to their design and place themselves in customer's shoes for a better understanding of customer needs (P2-1-1). Assume yourself as a customer like to experience the overall service process in person (P2-4-5) and by analogy (P1-4-2). 
(4) empathic creativity.

Dorothy and Rayport, (1997) also presented questions concerning empathic design for study, including in what way the implicit knowledge of end-users or designers can be revealed to understand how they find these needs and in what way these processes can be clearly analyzed for us to know the key requirements of potential customers. Nigel Cross, a major researcher representative, studies on designer-based issues like how to analyze and differentiate the implicit knowledge of designers such as innovative thinking.

Designers' thread of thought-thinking for a solution that may achieve the optimal effect-attraction (P1-5-2). Creative ways of designers include sitting still and emptying yourself first (P3-4-4) and rethinking (P3-4-5).

(5) empathic aesthetics.

The inner simulation and projected unity of object and subject of empathy imply the integration of the self and the object to become as one and generation of a variety of feelings. Empathy is required among the creator, the work, and the observer. Designers need to develop professional aesthetics.

As users do not necessarily have aesthetics, designers are required to meet the aesthetic need of users with professionalism (P3-5-3) and to satisfy proprietors with designers' beautification and incorporation (P3-8-5)

(6) empathic innovation.

Dorothy and Rayport (1997) proposed five steps in the process of empathic design to develop innovative results like IDEO. Whenever designers need to make a breakthrough for new ideas and new types (P2-8-8), they would think about how to have better and innovative performance during the re-designing process such as $a$ better purchase environment conceived by designers (P2-8-9). Designers need to apply their specialty to integrate and coordinate with the environment.

(7) empathic culture.

It is said recently that culture was the place of origin for empathy, the second social value. The human civilization would be impossible without this second value (Rifykin, 2001). Empathy, a bridge for mankind communication, helps establish a common view even among different cultures. Like Rifykin said, people sought after a common consensus through empathy, an ability of experiencing the feelings of others (Rifykin, 2001). The service quality is known by the brand corporate culture such as perfect customer satisfaction (p4-6-4). These habits, values and cultural backgrounds (P3-4-7) may set up a common view with customers (see Figure 3).

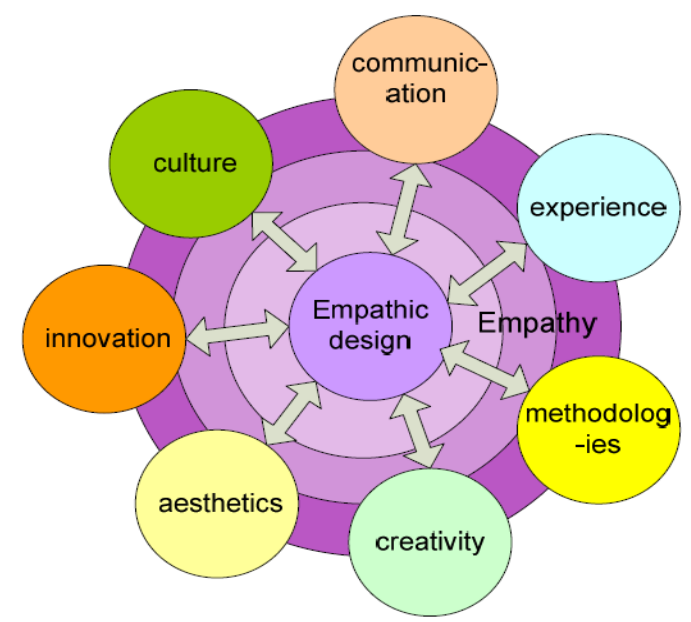

Figure 3. The seven dimensions of empathic design (this research illustration). 
Empathic design is an empathy-based design. There are seven dimensions inferred from the grounded theory and theories related to empathic design, including communication, experience, methodologies, creativity, aesthetics, innovation, and culture. All these seven dimensions can be studied independently and deeply. For instance, as far as communication is concerned, in addition to interpersonal communication between designers and their superiors, designers and users and designers and their proprietors, intangible communication and the brand spirit are also included. As for the dimension of experience, designers need to regard themselves as customers and experience the service process in person for a better understanding of customer perceptions. For methodologies, systematic ways are required for designers to help them be aware of user needs promptly. Designers may exercise these ways to develop originality, aesthetics, and innovative power and bring the product, service, and culture of the brand into existence in the end.

\section{Conclusion}

Empathic design is emerging in the field of design and developing from different perspectives in the east and the west. The thread of thought and the knowledge system of empathic design are constructed and based on philosophy, aesthetics, and psychology, etc. Accordingly, empathic design can not only be applied to design, but also to the process of creation, a communication medium, and experience of spatial display. The conclusions of this research are summarized as follows:

(1) Three of the four designers regard they accomplish customer satisfaction by experiencing in person and thinking in customer's shoes. They reason by analogy; i.e., they deem themselves as users and experience the overall purchase process so that they may obtain new ideas or modify from such experience.

(2) Thanks to experiences, designers may reduce mistakes. Designers also need to incorporate brand culture, background, and rules into design.

(3) Designers provide specialty, aesthetics, and satisfaction to serve users and users feel more satisfied when their position is the same as that of designers.

(4) The methods developed of empathy by role-play, reality practice, experience in person, scenario-oriented design, information of visual, storytelling, Mood board (images board), incorporation of requirements, sitting still with a peaceful mind and emptying ego and re-thinking etc.

(5) A common view can be reached through empathy in different cultures. Brands can obtain support and identification/recognition in different countries because of empathy.

(6) Seven dimensions of empathic design are discovered in this research from relevant theories, case analysis, and analysis of grounded theory, including communication, experience, methodologies, creativity, aesthetics, innovation, and culture. Related interdisciplinary researches extend continually such as inclusive design, healthcare, and wellbeing, etc. Independent in-depth researches are allowable for these seven dimensions.

(7) It is found empathy plays the most critical role for designers in communication, experience, methodologies, creativity, aesthetics, innovation, and culture.

\section{References}

Catherine, M., \& Rossman, B. G. (1995). Designing qualitative research (2th ed.). Newbury Park, CA: Sage. Chapman, J. (2005). Emotionally durable design: Objects, experiences and empathy. USA \& UK: Earthscan. Dorothy, L., \& Rayport, J. F. (1997). Spark innovation through emphatic design. Harvard Business Review, 75(6), 102. 
Feng, I., Lazar, J., \& Preece, J. (2004). Empathy and online interpersonal trust: A fragile relationship. Behaviour \& Information Technology, 23(2), 97-106.

Fritsch, J., Júdice, A., Soini, K., \& Tretten, P. (2007). Storytelling and repetitive narratives for design empathy: Case Suolinna. Retrieved from http://www.nordes.org

Herzog, T. (1996). Research methods and data analysis in the social sciences. New York: Addison.

Hogan, R. (1969). Development of an empathy scale. Journal of Consulting and Clinical Psychology, 33, 307-316.

Ickes, W. (1997). Empathic accuracy. New York: Guilford.

Kleef, E. V., Trijp, H. C., \& Luning, P. (2005). Consumer research in the early stages of new product development: A critical review of methods and techniques. Food Quality and Preference, 1(6),181-201.

Koskinen, I., Battarbee, K., \& Mattelmäki, T. (2003). Empathic design-User experience in product design. Finland: Edita.

Landwehr, P. (2007). Empathic design vs. empathetic design: A history of confusion. Retrieved from http://dataprivacylab.org/dataprivacy/projects/dialectics/designmethods/plandweh.pdf

Platt, J. (2002). The history of the interview. In F. J. Gubrium, \& A. J. Holstein (Eds.), Handbook of interview research: Context and method (pp. 33-54). London: Sage.

Rifkim, J. (2001). The age of access (p. 309). In Y. H. Huang (trs.). Taipei: Yuan-Liou.

Speroff, B. J. (1953). Empathy and role-reversal as factors in industrial harmony. Journal of Social Psychology, 37, 117-120. 